\title{
AVALIAÇÃO DA INFRAESTRUTURA DE TECNOLOGIA DA INFORMAÇÃO DE EMPRESAS EM MOSSORÓ-RN
}

\author{
Giovane Montine Moreira Gurgel \\ Professor do Instituto Federal de Educação, Ciência e Tecnologia do Rio Grande do Norte - \\ IFRN - Campus Mossoró. E-mail: giovane.gurgel@ifrn.edu.br \\ Paulo Cezar Filho \\ Aluno do Curso Técnico Subsequente em Sistemas de Informação do IFRN - Campus \\ Mossoró. E-mail: pauloczar10@ hotmail.com
}

\begin{abstract}
RESUMO
Este trabalho apresenta uma avaliação da infraestrutura de TI em organizações de diferentes ramos de atuação no município de Mossoró - RN. A pesquisa consiste na análise dos dados coletados via entrevista ao gerente de TI de cada organização. A análise comparou o percentual obtido em cada indicador com pesquisas nacionais sobre o uso de TI em empresas. Além dos indicadores nacionais, a pesquisa adotou o framework Control Objectives for Information and related Technology (Cobit) para identificar o nível de maturidade do processo AI3 - Adquirir e manter infraestutura de tecnologia. Os resultados demonstram que os gerentes de TI vêm conseguindo avançar com seus parques de informática. Mesmo com eventuais dificuldades de orçamento, foram encontrados vários serviços de infraestutura de TI com bons índices. A avaliação com base no Cobit revelou que $43 \%$ das empresas se encontram no nível 2 - "Repetível, porém intuitivo", 14\% se encontram no nível "Inicial/Ad hoc" e $14 \%$ com características do nível "Processo Definido". Ainda há muitos processos informais. Nenhuma empresa atendeu plenamente a algum nível de maturidade, é preciso aprimorar as práticas atuais. Novos estudos poderão analisar outros aspectos da infraestrutura de TI em grupos específicos de organizações ou até mesmo utilizando outros frameworks como o IT Infrastructure Library (ITIL).
\end{abstract}

PALAVRAS-CHAVE: tecnologia da informação, infraestrutura, gestão de TI

\section{ASSESSMENT OF INFRASTRUCTURE OF INFORMATION TECHNOLOGY OF COMPANIES AT MOSSORO-RN}

\begin{abstract}
This paper presents an assessment of IT infrastructure in organizations of different branches of activity in the Mossoró - RN. The investigation includes the analysis of data collected via an interview with the IT manager of each organization. The analysis compared the percentage obtained in each indicator to national surveys on the use of IT in enterprises. In addition to national indicators, the research adopted the framework Control Objectives for Information and related Technology (COBIT) to identify the level of process maturity AI3 - Acquire and maintain technology infrastructure. The results show that IT managers have been able to move forward with their information technology parks. Despite any difficulties in budget revealed several IT infrastructure services with a good index. The assessment based on Cobit revealed that $43 \%$ of firms at stage 2 - "Repeatable but intuitive, 14\% are at level" Initial ad hoc" and 14\% with some level characteristics "Defined". Still there are many informal processes. Neither company responded fully to some level of maturity, it is necessary to
\end{abstract}


improve current practices. Further studies should examine other aspects of IT infrastructure in specific groups of organizations or even using other frameworks like IT Infrastructure Library (ITIL).

KEYWORDS: information technology, infrastructure, IT management. 


\section{INFRAESTRUTURA DE TECNOLOGIA DA INFORMAÇÃO (TI): UMA} AVALIAÇÃO EM EMPRESAS DE MOSSORÓ-RN

\section{INTRODUÇÃO}

A Tecnologia da Informação (TI) tem papel importante no cenário competitivo das organizações, uma vez que torna seus processos mais ágeis e transparentes. Ao mesmo tempo, a TI vem trazendo também novos problemas em virtude do rápido crescimento do parque de computadores, sistemas, redes de comunicação, etc. Esse crescimento não-ordenado causa transtornos para os gestores de TI.

Dessa forma, surgem frameworks que tem como objetivo organizar a infraestrutura de TI das organizações. Semelhante às normas International Standards Organization (ISO), frameworks como o Control Objectives for Information and related Technology (CobiT), IT Infrastructure Library (ITIL), Project Management Body of Knowledge (PMBOK) e o Capability Maturity Model Integration (CMMI) orientam como os processos devem ocorrer para que tenham bons resultados.

Este trabalho faz parte do projeto de pesquisa "Avaliação da gestão de tecnologia da informação (TI) em empresas de Mossoró - RN" do IFRN - Campus Mossoró e tem como objetivo compreender o cenário atual da infraestrutura de TI de algumas empresas do município. A próxima seção trata do referencial teórico sobre a infraestrutura de TI e o framework CobiT. Em seguida, a metodologia utilizada é apresentada. Após, os resultados e a análise dos dados. A última seção traz as considerações finais, limitações e trabalhos futuros.

\section{REFERENCIAL TEÓRICO}

De acordo com Weill, Subramani e Broadbent (2002), 55\% do orçamento de TI das organizações é gasto com sua infraestrutura, envolvendo recursos humanos, tecnologia e processos. Assim, é necessário realizar um controle efetivo da infraestrutura para que as organizações possam obter vantagem competitiva a partir desses investimentos. Nesse sentido, a seção 2.1 identifica os componentes envolvidos na infraestrutura de TI. Em seguida, um framework de gerenciamento da TI - Cobit.

\section{INFRAESTRUTURA DE TI}

Os componentes da infraestrutura de TI estão representados na Figura 1 (WEILL; ROSS, 2006, p. 40). A infraestrutura suporta a demandas internas da organização (aplicações locais de TI), bem como realiza a interface com outras organizações externas (clientes, parceiros de negócios, infraestruturas públicas). 


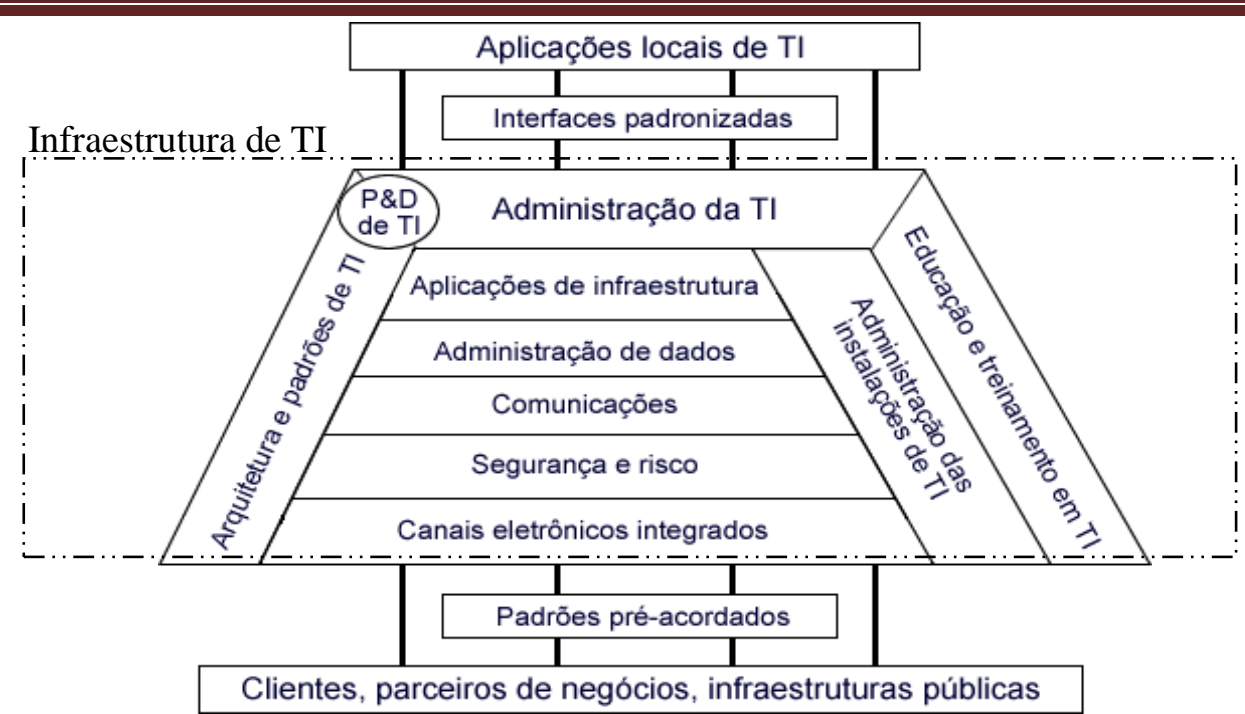

Figura 1 - Componentes da infraestrutura de TI.

Envolvendo tecnologia, processos e pessoas, uma das características principais da infraestrutura de TI é ser orientada a serviços modulares e configuráveis (DATTA et al., 2008). A visão da infraestrutura como serviço ajuda aos gestores a determinarem o valor da TI. Auxilia, por exemplo, na comparação de preços entre fornecedores. Do ponto de vista do fornecedor também é interessante uma vez que padroniza os serviços oferecidos independente das mudanças tecnológicas (WEILL; SUBRAMANI; BROADBENT, 2002).

No total, Weill, Subramani e Broadbent (2002) identificaram 70 serviços de infraestrutura de TI. Nesse sentido, cada um dos componentes (WEILL; ROSS, 2006) representa um subconjunto desses serviços. Os componentes foram agrupados em duas camadas: física e gestão. A camada física compreende os seguintes componentes:

- Canais eletrônicos integrados - serviços de comunicação com organizações externas através de diferentes canais de acesso;

- Segurança e risco - serviços de segurança e gestão de risco através de tecnologias como firewalls, políticas de controle de acesso remoto, uso de senhas, bem como o planejamento e a recuperação a desastres;

- Comunicações - serviços de comunicação dentro da própria organização e entre clientes e parceiros. Ocorrem através de interações eletrônicas por voz, vídeo, intranet, entre outras;

- Administração de dados - gestão dos dados de clientes, produtos e processos. Serviços garantem que informações críticas estejam disponíveis a pessoas e aplicações sempre que for necessário;

- Aplicações de infraestrutura - serviços utilizados por toda a organização (contabilidade, recursos humanos, orçamento, etc.). Por exemplo, o Enterprise Resource Planning (ERP) e o Customer Relationship Management (CRM). Possuem estreita relação com a "administração de dados";

- Administração das instalações de TI - serviços de gerenciamento da camada física. Por exemplo, processamento em larga-escala, ambientes de desenvolvimento, gerenciamento das aplicações, etc.; 
A camada de gestão compreende componentes que tratam da gestão de toda a infraestrutura de TI:

- Administração de TI - serviços de coordenação da infraestrutura integrada e gerenciamento de sua relação com as unidades de negócio. Inclui planejamento de sistemas de informação, gestão de projetos, acordos de nível de serviço (service level agreements - SLAs) e negociação com fornecedores;

- $\quad$ Arquitetura e padrões de TI - políticas no uso da TI em toda a organização. Os padrões são importantes ao levar em consideração a comunicação entre os diferentes atores na cadeia de valor. Os serviços provêem um plano de migração para outros padrões técnicos. Estão em constante desenvolvimento;

- Educação e treinamento em TI - frequentemente esquecida, a educação em tecnologias e sistemas da organização, bem como treinamento aos gestores de como investir e usar a TI para criar valor organizacional. Organizações que gastam mais em treinamento conseguem obter maior desempenho;

- Pesquisa e desenvolvimento de TI - interagindo com os grupos "administração de TI" e "arquiteturas e padrões de TI", busca novos meios de usar a TI para agregar valor.

As pesquisas demonstram uma correlação positiva entre a rapidez de implantação de novas iniciativas de negócio e a infraestrutura de TI. As organizações que administram a infraestrutura como um ativo e realizam investimentos com frequência, geralmente conseguem melhores resultados (WEILL; ROSS, 2006).

\section{FRAMEWORK COBIT}

A governança de TI tem como objetivo alinhar a TI aos requisitos do negócio. Para isso, as organizações utilizam frameworks que possuem um conjunto de práticas que permitem o alinhamento e o atendimento às estratégias do negócio através da TI. O CobiT contribui para o sucesso da entrega de produtos e serviços de TI ao organizar as atividades da TI em um modelo de processos genérico, identificando os principais recursos que precisam de maior investimento (FERNANDES; ABREU, 2008).

O CobiT demonstra como mensurar e como melhorar as práticas realizadas no ambiente de TI das organizações. Além de definir objetivos de controle que devem ser considerados pela gestão, o CobiT fornece o grau de maturidade em cada processo. Esses processos estão agrupados em quatro domínios, estabelecendo um modelo de gerenciamento da TI semelhante ao modelo tradicional de melhoria contínua (planejamento, execução, verificação e ação):

- Planejar e Organizar (PO) - trata das estratégicas e táticas ligadas ao uso da TI que contribuem para atingir os objetivos dos negócios. Auxilia a identificar se os riscos da TI são entendidos e gerenciados, se a organização está obtendo ótimo uso de seus recursos, entre outros;

- Adquirir e Implementar (AI) - para executar as estratégias, é preciso identificar soluções de TI, adquirindo ou desenvolvendo recursos que assegurem a continuidade dos negócios. Envolve ainda questões sobre o impacto da manutenção de sistemas e o tempo de entrega de novos projetos; 
- Entregar e Suportar (DS) - os processos relativos a esse domínio tratam da definição dos níveis de serviços requeridos, como: garantia de desempenho, segurança de sistemas, suporte aos serviços para usuários, gestão de dados e da infraestrutura operacional;

- Monitorar e Avaliar (ME) - define questões de auditoria, assegurando a qualidade dos serviços de TI. Trata basicamente de monitorar e avaliar o desempenho da TI, bem como verificar a aderência regulatória e do fornecimento da governança.

O CobiT compreende a infraestrutura de TI como recursos (hardware, sistemas operacionais, sistemas de gerenciamento de bases de dados, redes, entre outros) que possibilitam o processamento dos aplicativos. O processo AI3 (Quadro 1) - Adquirir e manter infraestrutura tecnológica - considera uma abordagem planejada para aquisição, manutenção e proteção da infraestrutura de TI. Assim, garante um apoio contínuo às aplicações de negócio (COBIT, 2007).

Quadro 1: Processo AI3 do Cobit - adquirir e manter infraestrutura de tecnologia.

Adquirir e manter infraestrutura tecnológica
que satisfaça aos seguintes requisitos do negócio para a TI:
adquirir e manter uma infraestrutura de TI integrada e padronizada
com foco em:
Disponibilizar plataformas apropriadas às aplicações de negócio em
alinhamento com a arquitetura de TI definida e os padrões tecnológicos
é alcançado por:
Preparação de um plano de aquisição tecnológica alinhado
com o plano de infraestrutura tecnológica
Planejamento da manutenção da infraestrutura
Implementação de controles internos, medidas de segurança e
de auditoria
e medido por:
Percentual das plataformas que não estejam alinhadas
com os padrões definidos de tecnologia e arquitetura de
TI
Quantidade de processos críticos de negócio
sustentados por infraestrutura obsoleta (ou próxima da
obsolescência)
Quantidade de componentes de infraestrutura que não
contam mais com suporte (ou que tendem a não ter
suporte num futuro próximo)

FONTE: Cobit (2007, p. 83).

Entre os objetivos de controle do processo AI3: a preparação de um plano de aquisição; garantia de auditabilidade, disponibilidade e integridade; desenvolvimento de um plano de manutenção e o estabelecimento de um ambiente de testes para novos componentes da infraestrutura. Dessa forma, é garantido o fornecimento de uma infraestrutura de TI segura e confiável (COBIT, 2007). O modelo de maturidade do processo AI3 (Tabela 1) permite mensurar o desempenho e identificar melhorias necessárias. 
Tabela 1: Níveis de aquisição e manutenção da infraestrutura de TI.

\begin{tabular}{|c|c|}
\hline Nível & Descrição \\
\hline 0 - Inexistente & $\begin{array}{l}\text { O gerenciamento da infraestrutura tecnológica não é } \\
\text { reconhecido como um tópico suficientemente } \\
\text { importante para ser considerado }\end{array}$ \\
\hline 1 - Inicial/ Ad Hoc & $\begin{array}{l}\text { Infraestrutura é importante, embora não seja } \\
\text { abordada de forma consistente }\end{array}$ \\
\hline 2 - Repetitivo, mas intuitivo & $\begin{array}{l}\text { Aquisiçãa e manutenção da infraestrutura de TI não } \\
\text { são baseadas em uma estratégia definida }\end{array}$ \\
\hline 3 - Processo Definido & $\begin{array}{l}\text { A manutenção da infraestrutura de TI é planejada, } \\
\text { programada e coordenada }\end{array}$ \\
\hline 4 - Gerenciado e mensurável & $\begin{array}{l}\text { Aquisição e manutenção da infraestrutura de TI } \\
\text { funcionam bem na maioria das situações, é bem } \\
\text { organizado e dinâmico }\end{array}$ \\
\hline 5 - Otimizado & $\begin{array}{l}\text { A infraestrutura de TI é visto como fator decisivo, } \\
\text { os custos são reduzidos através da racionalização e } \\
\text { padronização da infraestrutura }\end{array}$ \\
\hline
\end{tabular}

A capacidade de avaliar processos permite às organizações tomar melhores decisões sobre a infraestrutura de TI. O custo elevado para manter os equipamentos, faz com que muitas organizações contratem fornecedores especializados que proporcionam serviços de hardware e software superiores ao que as organizações conseguiriam manter em seus departamentos de TI (DUENING; CLICK, 2005).

Portanto, uma infraestrutura de TI bem projetada e modular proporciona uma maior flexibilidade nas aplicações de TI, resultando em melhores taxas de retorno sobre o investimento, além da rápida implantação de novas aplicações (LIU, 2002). É importante ressaltar que a infraestrutura hoje é uma combinação de infraestrutura privada (unidade de negócio, organização, setor de atuação) e pública (Internet), logo, as opções são muitas e as decisões precisam ser criteriosas (VERAS, 2009).

\section{METODOLOGIA}

Essa pesquisa consiste de um estudo descritivo já que busca descrever os fatos e fenômenos de determinada realidade (TRIVIÑOS, 2008). A infraestrutura de TI de organizações é investigada e avaliada com base em modelos de referência.

O levantamento dos dados será realizado em diversas empresas no município de Mossoró RN a partir de entrevista in loco ao gestor de TI de cada empresa. Foram entrevistados sete gerentes de TI. O roteiro de entrevista é composto por 21 questões - quatro abertas e 17 fechadas - envolvendo várias camadas da infraestrutura de TI relatados por Weill, Subramani e Broadbent (2002). Outro modelo de referência utilizado foi o Cobit. Como o foco da pesquisa era a infraestrutura de TI, optou-se por avaliar o grau de maturidade das empresas no processo AI3 - adquirir e manter infraestrutura de tecnologia.

Apesar das características de pesquisa qualitativa, Creswell (2007) ressalta que vários métodos de investigação podem ser utilizados. Nesse sentido, a análise dos dados será realizada através de estatística descritiva e a interpretação dos dados coletados. Muitas questões foram utilizadas em outras pesquisas, como a "Pesquisa sobre o uso das tecnologias da informação e da comunicação no Brasil: TIC Domicílios e TIC Empresas 2009" realizada pelo Comitê Gestor da Internet no Brasil. Isso permite a comparação com os indicadores 
nacionais. A observação também foi utilizada no momento da entrevista onde geralmente se encontrava todo o parque de infraestrutura de TI da empresa. A partir da observação e das respostas obtidas nos questionários, foi identificado o nível de maturidade das organizações no processo $\mathrm{AI} 3$ do framework CobiT.

\section{ANÁLISE DOS RESULTADOS}

Foram realizadas entrevistas ao responsável pela TI em sete empresas. Apenas em uma empresa foi utilizada outra nomenclatura para o cargo de gerente de TI. As equipes de TI geralmente eram compostas por, no máximo, quatro pessoas. Todos os respondentes eram do sexo masculino e o tempo médio que ocupavam o cargo de gerente de TI nas empresas foi de 8 anos.

As empresas possuíam, em média, 81 computadores. Ao levar em conta os índices nacionais, as empresas pesquisadas se encontram no grupo das $9 \%$ que possuem mais de 31 computadores na região nordeste. $\mathrm{O}$ mercado de atuação de cada empresa foi distinto, entre eles: serviços, indústria de transformação, comércio, etc. A Tabela 2 demonstra o resultado obtido em nove itens do questionário sobre a infraestrutura de TI. As opções disponíveis para resposta eram "SIM" ou "NÃO".

Tabela 2: Proporção dos indicadores da infraestrutura de TI nas empresas pesquisadas.

\begin{tabular}{|c|c|c|}
\hline Questão & SIM & $\begin{array}{c}\mathrm{NA} \\
\mathrm{O}\end{array}$ \\
\hline Sua empresa possui uma LAN/Rede cabeada? & $100 \%$ & $0 \%$ \\
\hline Sua empresa possui uma LAN/ Rede sem fio? & $86 \%$ & $14 \%$ \\
\hline Sua empresa possui a intranet? & $29 \%$ & $71 \%$ \\
\hline Sua empresa possui um software ERP? & $71 \%$ & $29 \%$ \\
\hline Sua empresa possui um software CRM? & $14 \%$ & $86 \%$ \\
\hline Sua empresa utiliza algum sistema operacional (SO) gratuito nos servidores? & $43 \%$ & $57 \%$ \\
\hline Sua empresa utiliza algum SO gratuito nos computadores de usuários finais? & $29 \%$ & $71 \%$ \\
\hline Sua empresa possui um website? & $71 \%$ & $29 \%$ \\
\hline Sua empresa possui uma política de segurança de informação? & $71 \%$ & $29 \%$ \\
\hline
\end{tabular}

Acima da média nacional de $79 \%$ das empresas da região nordeste, ficou constatado que em todas as empresas havia uma rede local cabeada. No entanto, $86 \%$ das empresas possuíam rede sem fio. Mesmo assim é um resultado acima da média de $40 \%$ das empresas do nordeste com rede sem fio. O resultado sobre a questão da intranet - $29 \%$ - ficou um pouco acima da média da região nordeste $-23 \%$. Ao perguntar sobre o software ERP, o resultado $-71 \%$ - foi acima dos $25 \%$ da região nordeste. Foi identificada a presença de grandes fornecedores de pacotes ERP como a Totvs e a SAP. Já sobre o software Customer Relationship Management (CRM) foi obtido um valor $-14 \%$ - abaixo da média da região nordeste $-31 \%$. Vale salientar que nem sempre os softwares disponíveis nas empresas eram específicos para a atividade de CRM. Geralmente alguns módulos eram utilizados para realizar alguma tarefa sobre a base de dados dos clientes. Inclusive, às vezes, sem auxílio de software. Em alguns casos as empresas utilizavam de trabalho manual para realizar as atividades que uma aplicação CRM pode oferecer.

Sobre o uso de SO gratuito nos servidores, apenas $43 \%$ das empresas pesquisadas fazem uso. É um resultado inferior à média da região nordeste - $58 \%$ - e próximo ao da região sul do Brasil - 47\%. Já o resultado para o uso de SO gratuito nos computadores de usuários finais $29 \%$ - foi maior do que a média da região nordeste - $13 \%$. Apesar da proporção menor de SO 
gratuito no servidor, vale salientar que nem sempre as empresas possuem a licença para uso de software proprietário. Acima da média do nordeste $-40 \%, 71 \%$ das empresas pesquisadas possuíam um website. A política de segurança de informação é considerada por $71 \%$ das empresas pesquisadas. Essa proporção está acima das médias do nordeste. A pesquisa revelou que alguns procedimentos de segurança de informação são postos em prática, mas não são totalmente documentados.

A Tabela 3 apresenta a proporção dos tipos de conexão que as empresas utilizavam para acessar à Internet. Nas estatísticas da região nordeste, a conexão mais típica é a via Digital Subscriber Line (DSL). Isso também foi constatado na pesquisa. A conexão via linha dedicada ainda é utilizada por empresas de Mossoró, são linhas que possuem um maior custo operacional, mas com maior garantia de disponibilidade. Alguns gestores informaram que a conexão via DSL tem causado eventuais transtornos como a queda do link e a lentidão no acesso à Internet. Algumas alternativas já estão sendo avaliadas como a conexão de banda larga via TV a CABO. Como é um serviço recente sendo oferecido na cidade, as empresas ainda estão se adaptando e analisando essa nova alternativa de conexão à Internet. Os links de acesso encontrados nas empresas geralmente eram de um ou dois Mbps (Megabit por segundo). A redundância não estava presente em todas as empresas, no entanto, os gestores ressaltavam a importância do procedimento e muitos informaram que já estavam trabalhando para adquirir novos links ou adequar a infraestrutura atual para oferecer maior disponibilidade no acesso à Internet a partir da empresa.

Tabela 3: Tipo de acesso à Internet.

\begin{tabular}{|c|c|}
\hline DSL & $57 \%$ \\
\hline RÁDIO & $29 \%$ \\
\hline LINK DEDICADO & $43 \%$ \\
\hline
\end{tabular}

As questões observadas até o momento estão relacionadas aos serviços de infraestrutura da camada física segundo Weill, Subramani e Broadbent (2002). Ao analisar itens relacionados à camada de gestão, é preciso avaliar, por exemplo, se a arquitetura de TI está adequada às necessidades do negócio e sem esquecer o lado humano da tecnologia. Treinamentos freqüentes para a equipe são importantes e não apenas para a equipe de TI, mas para os outros gerentes também, afinal de contas eles precisam compreender o potencial da informática para poder auxiliar nas decisões que precisam tomar em favor da organização. A pesquisa "TIC Empresas 2009" demonstra dados sobre a contratação de especialistas em TI no Brasil. Nesse sentido, foram questionados ao gestor de TI quais os principais motivos que, no momento da seleção de novos funcionários, impediram que o candidato fosse efetivado no cargo. Cerca de $29 \%$ dos gestores afirmaram que o fator que impediu a contratação foi a falta de experiência na atividade desejada. Em torno de $43 \%$ apontaram a falta de candidato e $43 \%$ consideraram que o candidato não tinha qualificação necessária para o cargo. Os dados apontam que há poucos profissionais da área de TI na cidade e muitos precisam melhorar sua qualificação e experiência da prática profissional. É uma situação até melhor se observar os índices da região nordeste que estão acima de $51 \%$ para as três questões.

A gestão da TI considera a infraestrutura e os requisitos de negócio. O processo AI3 do framework Cobit trata do planejamento, aquisição e manutenção da infraestrutura de TI nas organizações. No levantamento realizado, foi questionado a cada gestor de TI sobre como esse processo é realizado nas suas empresas. Alguns pontos como a infraestrutura que mantém as aplicações críticas, as plataformas existentes e a existência de um plano de manutenção foram considerados muito importantes pelos gestores de TI. Os gestores também consideraram a infraestrutura como a parte da TI que demanda grandes investimentos. A maior parte dos gestores $-86 \%$, afirmaram que suas aplicações críticas encontram-se em uma 
infraestrutura atualizada. Mesmo com dificuldades de recursos, o que foi percebido é que eles vem conseguindo obter, à medida do possível, aquilo que precisam para implementar as ações do departamento de TI em cada empresa. No entanto, ainda há empresas onde a TI não está inserida em seu planejamento anual, ou seja, as ações são muito reativas. Por exemplo, um dos gestores deixou claro que boa parte dos investimentos ocorria em virtude de uma nova legislação que a empresa precisava atender. Cerca de 57\% dos gestores afirmaram que há variedade de plataformas tecnológicas em seu ambiente de TI. Isso impacta diretamente na manutenção. Cerca de $43 \%$ dos gestores afirmaram que fazem um plano de manutenção. É realizado um planejamento junto ao planejamento geral da empresa ou é feito um cronograma onde os equipamentos de TI são acompanhados e monitorados a fim de prevenir alguma falha com o decorrer do tempo de seu uso. Outro ponto importante na classificação de uma organização quanto ao nível de maturidade no AI3 do Cobit é a existência de um ambiente de testes a fim de promover testes de viabilidade e integração à infraestrutura. Em todas as empresas pesquisadas foi identificado que havia um ambiente para realizar testes de novas tecnologias antes de serem entregues aos usuários finais. Assim, a partir desses dados e da observação realizada em cada organização, é possível afirmar que nenhuma das empresas está no nível 0 - "Inexistente". Todas entendem que a infraestrutura é importante, mas nenhuma atende plenamente aos requisitos propostos nos níveis 2 - "Repetível, porém intuitivo" ou 3 "Processo Definido". Apenas 14\% das empresas mais se aproximam do nível 3 - "Processo Definido", mas ainda falha em alguns pontos como a disponibilidade e um plano formal para adquirir, implementar e manter a infraestrutura de TI. Outros 14\% das empresas se encontram no nível "Inicial/Ad hoc" e os $43 \%$ restantes no nível 2 - "Repetível, porém intuitivo".

\section{CONCLUSÃO}

A maioria dos responsáveis pelo setor de TI ocupava o cargo de gerente de TI. Isso representa uma padronização que até a pouco tempo não existia. Na verdade, ainda há muitas empresas que não atribuem a nomenclatura correta na tentativa de pagar salários não compatíveis aos do nível gerencial. Foi constatado em todas as empresas que, mesmo com dificuldades, os gerentes de TI vêm conseguindo avançar em seus parques de informática. A partir da observação da infraestrutura, ficou claro que o alto escalão de muitas empresas reconhece o valor da TI para seus negócios. Isso é muito positivo, mas ainda há muitos "cortes" ao se falar em investimentos para a área. Uma das forças que possibilita o investimento ainda é o atendimento a legislações; nesses casos, ou investe ou será penalizado. Em quase todos os aspectos investigados na infraestrutura de TI, as empresas de Mossoró - RN demonstraram índices maiores do que a média da região nordeste, às vezes próximo até de índices de regiões mais avançadas como o sudeste e sul do Brasil. Apesar do levantamento realizado não abranger todos os serviços de infraestrutura identificados por Weill, Subramani e Broadbent (2002), a pesquisa revelou que as organizações não implantavam muitos processos das camadas de gestão e física.

De modo similar, muito pouco do framework Cobit foi identificado nas empresas. Elas estão avançando, mas precisam aprimorar suas práticas atuais. Percebe-se que ainda há muitos processos informais. As empresas podem avançar de nível no processo AI3 e com poucas ações, a maioria delas podem consolidar o nível 2 - "Repetível, porém intuitivo". Vale salientar que a busca por maiores níveis só traz benefícios para as organizações, quanto maior o nível, melhores resultados obtidos com o uso de TI.

Uma limitação desta pesquisa é não ter analisado a terceirização ou outros aspectos mais detalhados que estão relacionados com a infraestrutura de TI. Apesar disso, outros estudos poderão complementar essa análise ao focar em outros temas. Essa pesquisa analisou empresas de diferentes ramos de atuação. Novas pesquisas poderão verificar a infraestrutura 
de TI de organizações de um ramo ou determinado porte. Novas pesquisas poderão analisar também outros processos do Cobit, ou até mesmo utilizar outros frameworks como o ITIL.

\section{REFERÊNCIAS}

1. CRESWELL, J. W. Projeto de pesquisa: métodos qualitativo, quantitativo e misto. 2ed. Porto Alegre: Artmed, 2007. 248p.

2. DATTA, P.; TROUTT, M.; BOOTH, D.; SHANKER, M. A service-design of it infrastructure. 39th Annual Meeting of the Decision Sciences Institute. pp. 34813486, 22-25 nov. 2008.

3. DUENING, T. N.; CLICK, R. L. Essentials of business process outsourcing. Hoboken: John Wiley \& Sons, Inc, 2005.

4. FERNANDES, A. A.; ABREU, V. F. Implantando a governança de TI. Rio de Janeiro: Brasport, 2008. 480p.

5. IT GOVERNANCE INSTITUTE. COBIT 4.1: Framework Control Objectives Management Guidelines Maturity Models. Rolling Meadows, IL, 2007.

6. LIU, S. A Practical Framework for Discussing IT Infrastructure. IT Professional, v. 4, n. 4, pp. 14-21, July/Aug. 2002.

7. Pesquisa sobre o Uso das Tecnologias da Informação e da Comunicação no Brasil : TIC Domicílios e TIC Empresas 2009 [coordenação executiva e editorial, Alexandre F. Barbosa]. São Paulo: Comitê Gestor da Internet no Brasil, 2010.

8. TRIVIÑOS, A. N. S. Introdução à pesquisa em ciências sociais: a pesquisa qualitativa em educação. São Paulo: Atlas, 2008. 175 p.

9. VERAS, M. Datacenter: componente central da infraestrutura de TI. Rio de Janeiro: Brasport, 2009. 376p.

10. WEILL, P.; ROSS, J. W. Governança de Tecnologia da Informação. São Paulo: M. Books, 2006. 274 p.

11. WEILL, P.; SUBRAMANI, M.; BROADBENT, M. Building IT Infrastructure for Strategic Agility. MIT Sloan Management Review, v. 44, n. 1, Fall, 2002. 